\title{
openheart Prevalence and prognostic value of echocardiographic screening for rheumatic heart disease
}

\author{
Susy Kotit, ${ }^{1,2,3}$ Karim Said, ${ }^{2,4}$ Amr ElFaramawy, ${ }^{2,4}$ Hani Mahmoud, ${ }^{2}$ \\ David I W Phillips, ${ }^{5}$ Magdi H Yacoub ${ }^{1,2,3}$
}

To cite: Kotit S, Said K, EIFaramawy A, et al. Prevalence and prognostic value of echocardiographic screening for rheumatic heart disease. Open Heart 2017;4:e000702. doi:10.1136/

openhrt-2017-000702

Received 21 August 2017

Revised 24 August 2017

Accepted 7 November 2017

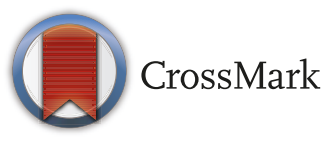

${ }^{1}$ National Heart and Lung Institute, Imperial College, London, UK

${ }^{2}$ Aswan Heart Centre, Aswan, Egypt

${ }^{3}$ Harefield Heart Science Centre, Imperial College London, London, UK

${ }^{4}$ Cardiology Department, Kasr El Ainy Faculty of Medicine, Cairo University, Cairo, Egypt ${ }^{5}$ Developmental Origins of Health and Disease Division, University of Southampton, Southampton General Hospital, Southampton, UK

Correspondence to Dr Susy Kotit, Aswan Heart Centre, Aswan, Egypt; susykotit@hotmail.com

\section{ABSTRACT}

Objective Rheumatic heart disease (RHD) remains a major health problem in many low-income and middleincome countries. The use of echocardiographic imaging suggests that subclinical disease is far more widespread than previously appreciated, but little is known as to how these mild forms of RHD progress. We have determined the prevalence of subclinical RHD in a large group of schoolchildren in Aswan, Egypt and have evaluated its subsequent progression.

Methods Echocardiographic screening was performed on 3062 randomly selected schoolchildren, aged $5-15$ years, in Aswan, Egypt. Follow-up of children with a definite or borderline diagnosis of RHD was carried out 48-60 months later to determine how the valvular abnormalities altered and to evaluate the factors influencing progression. Results Sixty children were initially diagnosed with definite RHD (19.6 per 1000 children) and 35 with borderline disease (11.4 per 1000); most had mitral valve disease. Of the 72 children followed up progression was documented in 14 children (19.4\%) and regression in 30 $(41.7 \%)$ children. Boys had lower rates of progression while older children had lower rates of regression. Functional defects of the valve even in the presence of structural features were associated with lower rates of progression and higher rates of regression than structural changes.

Conclusions RHD has a high prevalence in Egypt. Although a high proportion of the abnormalities originally detected persisted at follow-up, both progression and regression of valve lesions were demonstrated.

\section{INTRODUCTION}

Rheumatic heart disease (RHD) remains a major health problem in many low-income and middle-income countries. ${ }^{1-4}$ While it is estimated that 33 million people suffer from RHD worldwide, ${ }^{5}$ this is likely to be an underestimate due to the presence of undiagnosed RHD ${ }^{6}$ and the paucity of systematic screening studies in high-risk areas. Transthoracic echocardiography is a sensitive and specific screening tool which enhances the detection rate of RHD by a factor of up to tenfold. ${ }^{7-9}$ Studies based on echocardiography have reported prevalence rates of RHD which vary widely, from 15 per 1000 in Northern

\section{KEY QUESTIONS}

What is already known about this subject? The true prevalence of rheumatic heart disease (RHD) is underestimated in surveys due to the presence of mild forms of the disease which can only be detected by targeted echocardiography using standardised diagnostic criteria. Little is known as to how these subclinical lesions progress.

What does this study add?

This study provides an up to date estimate of the prevalence of RHD in a large group of schoolchildren in Aswan using current diagnostic criteria. It also provides information as to how the echocardiographic findings change over a 48-60 month period.

How might this impact on clinical practice? These data on the prevalence and outcome of subclinical RHD provide important information for the planning of cardiology services, highlight the need for RHD registers and the initiation of a multisectoral and integrated national RHD programme to achieve secondary prevention with penicillin prophylaxis.

Australia $^{410}$ to 48 per 1000 in Nicaragua. ${ }^{11}$ However, differences in the diagnostic criteria and interpretation of the images used greatly affect the reported prevalence rates ${ }^{12}$ and have led to the development of standardised World Heart Federation (WHF) criteria for the interpretation of echocardiograms. These have been widely adopted and are currently regarded as the gold standard. ${ }^{6}$ A still largely unanswered question, however, is the extent to which subclinical lesions detected by echocardiography are important in predicting significant cardiac disease. ${ }^{4} 8$ 13-16

Because there are no data on the prevalence of RHD in Egypt using the current WHF criteria, we have determined its prevalence in a large group of schoolchildren in Aswan. We have also evaluated how the subclinical findings detected by echocardiography progress during the subsequent $4-5$ years. 

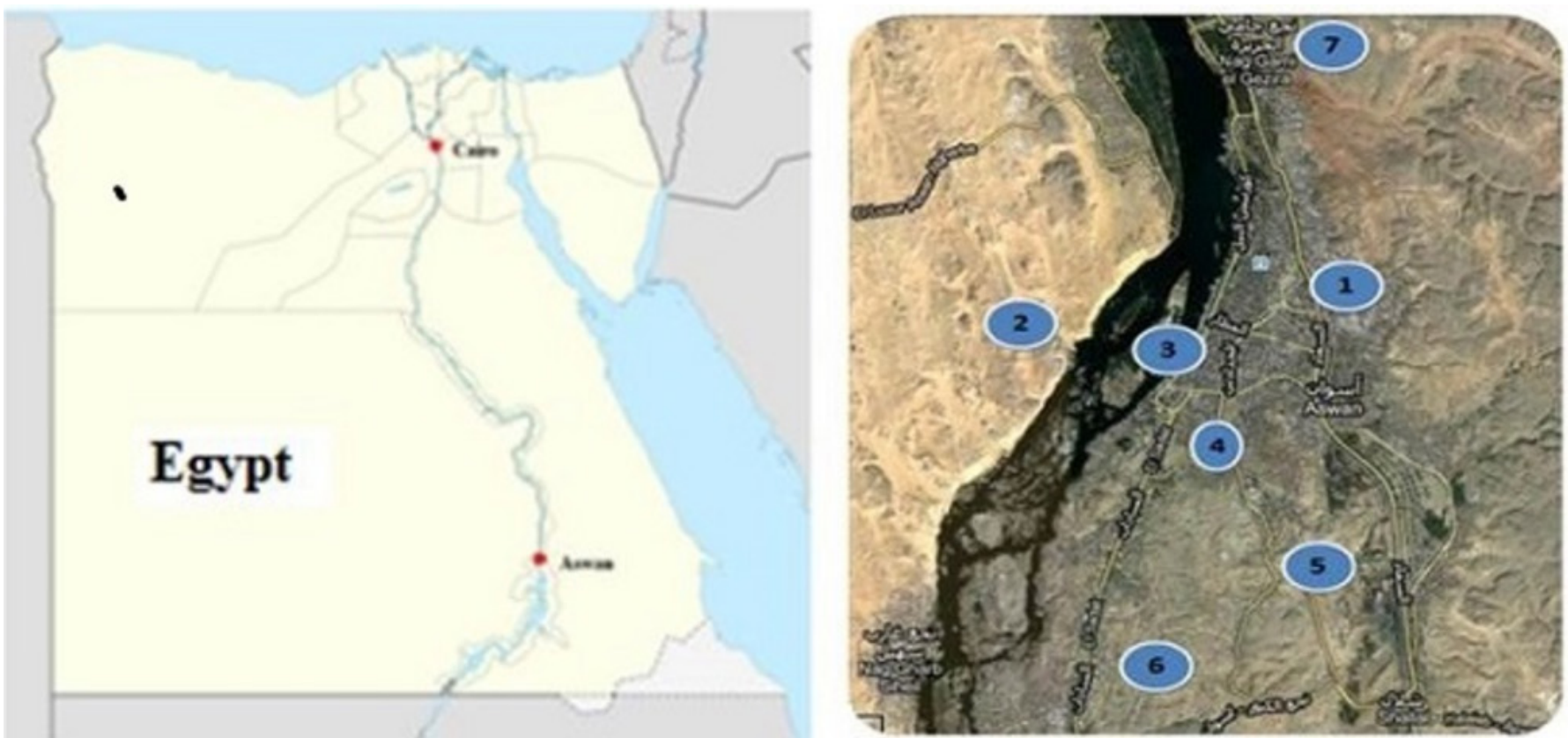

Figure 1 Location of Aswan, Egypt, and the city's seven geographical areas.

\section{METHODS}

\section{Study population and sample selection}

The Governorate of Aswan consists of seven geographical areas (figure 1) comprising a population of 1431488 people. An age-stratified random sample of children was selected from the schools within these areas with the aim of screening a minimum of 3000 subjects, about $6 \%$ of all children in Aswan. Because the schools admitted children in three different age groups (5, 6-12 and 13-15years), the sample size within each school was weighted in proportion to the number of children in each age group and the size of each survey area such that each child had the same probability of being selected. The initial screening was started in September 2009 with follow-up visits at intervals of 6 months. The analysis includes data up to the last follow-up carried out in September 2014.

\section{Examination protocol}

The children were asked about their medical history and cardiac auscultation was carried out with the patient in the supine and left lateral decubitus positions for the detection of any heart murmur. Detailed transthoracic echocardiography was performed with the use of a hand-carried echocardiographic system (General Electric Vivid I, Milwaukee, Wisconsin, USA) with M-mode, two-dimensional and Doppler (pulsed, continuous and colour) imaging. The following views were obtained: parasternal long-axis and short-axis views at the level of the papillary muscles and the aortic valve (AV), apical two-chamber, four-chamber and five-chamber views and subcostal views. Particular attention was paid to valve morphology on cross-sectional, two-dimensional imaging and function assessed by colour flow Doppler imaging. The apical imaging included anterior angulation to evaluate the left ventricular outflow tract and the AV.
Transvalvular flow was assessed by measuring the peak velocity with continuous wave Doppler imaging. All examinations were performed by a physician researcher (SK) trained in echocardiography and reviewed by two senior cardiologists unaware of the initial diagnosis. Echocardiograms were evaluated based on the WHF criteria which define criteria for the diagnosis of 'definite' and 'borderline' RHD. ${ }^{6}$ Where there was discordance between the original diagnosis and diagnosis following review, subjects were invited to the Aswan Heart Centre (AHC) for repeat echocardiograms and the final categorisation of RHD status was based on the consensus between the two senior cardiologists and the researcher.

All definite and borderline cases were asked to participate in the follow-up examinations. In addition, a control for each case was selected and matched for age ( \pm 1 year) and gender from the echocardiographically normal group. Follow-up echocardiograms were carried out using the same criteria and procedures as the initial study. Progression of valvular lesions was defined as development of a new structural or functional abnormality or progression of severity of a functional valve lesion (regurgitation/stenosis) based on the American Heart Association/ American College of Cardiology (AHA/ACC) valvular heart disease criteria for severity. Regression of valvular lesions was defined as disappearance of a structural or functional abnormality or decrease of severity of a functional valve lesion (regurgitation/stenosis) based on the same criteria as progression.

The factors analysed for their influence on the clinical outcome of subclinical RHD included age, gender, compliance with penicillin prophylaxis, type of pathology found at the baseline examination, presence of functional or structural defects, presence or absence 
of MR and time interval between the examinations. Prophylaxis compliance was assessed by reviewing the medical records reporting dates of administration of the injections.

\section{Ethics}

The research project was approved by the appropriate authorities at the Governorate of Aswan and the Egyptian Ministry of Health and Education, as representatives of the population and education system. The local authorities and school personnel were given written and oral detailed information and were notified about the results of the tests and eventual need of any further treatment or follow-up. Children with cardiac disease were offered free treatment and the recommendation for initiation of the penicillin prophylaxis, follow-up and surgery if indicated at the AHC. Because the history of rheumatic fever was unknown and we could not, being in an endemic/ high risk area, exclude previous episodes, all definite and borderline cases were started on prophylactic penicillin as secondary prophylaxis has been documented to reduce significantly the risk of recurrent attacks and morbidity and mortality related to the condition.

\section{Statistical analysis}

Based on a prevalence of RHD of between $2.5 \%$ and $3.0 \%$ as determined by previous echocardiographic studies of schoolchildren, ${ }^{8-11} 17$ we estimated that a sample size of a minimum of 3000 children would enable us to estimate the prevalence with a SE of approximately $0.3 \%$.

Data were analysed using Statistical Package for Social Sciences, V.16 (SPSS 16). Descriptive data are presented as number (n) and per cent (\%) and mean \pm SD; sample means were compared with an independent-sample Student's t-test. Bivariate analysis of outcome measures was undertaken comparing all cases with controls. Fisher's exact test was used where the sample sizes were small. A $\mathrm{P}$ value $<0.05$ was taken to indicate statistical significance and all tests were two-sided.

\section{RESULTS}

The mean age of the 3062 children was 10.0 years ( $\mathrm{SD} \pm 2.6$, range $5-15$ years), with a slight male predominance $(58.7 \%, \mathrm{n}=1796)$. None of the children were known to have cardiac disease and none were taking penicillin prophylaxis. Of the 63 children originally classified as showing definite disease by the researcher 58 were confirmed on review as were 23 of the 26 children with borderline disease. Among the 2967 normal studies, 7 were considered to be borderline on review. Following re-evaluation of the discordant cases, a total of 95 children were considered to have RHD (31.0 per 1000 children). Sixty had definite RHD (19.6 casesper 1000 children) and afurther 35 borderline disease (11.4 cases per 1000). Among the 60 cases of definite disease, 57 had mitral valve (MV) disease, 2 had disease of the AV and 1 child had borderline disease of both the $\mathrm{AV}$ and MV. Of the 35 children with borderline disease,
12 had two or more morphological features of mitral disease, 22 had mitral and 1 had aortic regurgitation.

Five $(7.1 \%)$ of the 60 children diagnosed with definite RHD by echocardiography had a pansystolic murmur on auscultation while 2 of the 3002 echocardiographically normal children had murmurs-giving a sensitivity for auscultation of $8.3 \%$, a specificity of $99 \%$ and positive predictive value of $71 \%$. Three children had congenital heart disease (prevalence 1 per 1000), with one case each of patent ductus arteriosus, partial atrioventricular canal and congenitally corrected transposition of the great arteries.

Of the 95 children diagnosed with borderline or definite RHD, 72 (75.8\%) were followed up an average of 42.1 ( $\mathrm{SD} \pm 12.9$, range $18-60$ ) months after the original survey. This group comprised 46 children with an original diagnosis of definite RHD and 26 with borderline RHD. There were 38 boys and 34 girls with a mean age of 14.1 ( $\mathrm{SD} \pm 2.63$ ) years. The majority of the children were compliant with the penicillin prophylaxis $(n=60$, $83.3 \%)$. The remaining children diagnosed at the time of the screening refused follow-up. Controls who had no pathology on the baseline echocardiogram consisted of 59 boys and 21 girls with a mean age of $15.74(\mathrm{SD} \pm 2.34)$ years, followed up a mean of 49.7 ( $\mathrm{SD} \pm 4.15$, range $18-60$ ) months after the initial screening.

Of the 46 definite cases, 9 had regressed such that there was no evidence of disease, 4 had become borderline and 33 continued to have definite disease. Ten of the borderline cases had become normal, 10 were unchanged and 6 had progressed to develop definite RHD. Among the 80 controls, none developed evidence of RHD according to the WHF classification.

Table 1 shows the detailed nature of the changes in echocardiographic appearance within each of the diagnostic groups based on the actual change of severity of the lesions, regardless whether the change lead to an alteration in the WHF grading of disease. Among the 46 definite cases, 18 were unchanged and 8 showed evidence of disease progression as evidenced by an increase in functional severity. Among the 20 who had regressed, 9 were completely disease-free on follow-up and of the 11 who continued to have some evidence of RHD, 9 showed a reduction in functional severity and 2 an improvement in the structural changes. Among the six borderline cases that had progressed, four had evidence of increased functional severity and two showed increased structural changes. Of the 10 who regressed, 7 showed evidence of reduced functional severity and 3 had regression of structural changes. In the control group, valvular thickening appeared in seven children.

Further analyses were carried out to assess the factors related to progression or regression of the echocardiographic changes. Among the 14 children who progressed, boys tended to have a lower rate of progression than girls, $5(13 \%)$ of 38 boys versus 9 (26\%) of 34 girls. There was strong evidence of lower rates of regression of valve lesions in the older children (regression was seen in $23(77 \%)$ of 
Table 1 Functional and structural changes underlying the progression or regression of the cardiac abnormalities in the children with definite or borderline disease at initial screening compared with the control group

\begin{tabular}{|c|c|c|c|}
\hline \multirow[b]{2}{*}{ Follow-up status } & \multicolumn{3}{|c|}{ Initial RHD category } \\
\hline & Definite $(n=46)$ & Borderline $(n=26)$ & Control $(n=80)$ \\
\hline Unchanged & $18(39.1 \%)$ & $10(38.5 \%)$ & $73(91.2 \%)$ \\
\hline Progression & $8(17.4 \%)$ & $6(23.1 \%)$ & $7(8.8 \%)$ \\
\hline Increase in functional severity & 8 & 4 & 0 \\
\hline Appearance of structural changes & 0 & 2 & 7 \\
\hline Regression & $20(43.5 \%)$ & $10(38.5 \%)$ & \\
\hline Resolution of functional and structural abnormalities & 9 & 0 & \\
\hline Reduction in functional severity & 9 & 7 & \\
\hline Regression of structural changes & 2 & 3 & \\
\hline
\end{tabular}

RHD, rheumatic heart disease.

30 children studied at the age of 10-15 years compared with only $7(23 \%)$ of 30 in the age group 16-19 years, $\mathrm{P}<0.001)$. This was not related to initial severity of the lesion, as all lesions seen in this study were mild and had similar severity at the initial screening. The follow-up time was not significantly correlated to progression or regression of disease. High rates of compliance with penicillin prophylaxis were achieved $(83 \%$ of definite and $85 \%$ of borderline cases), and although there was a trend towards lower progression in compliant compared with non-compliant subjects, 14 (23\%) of 60 children versus 6 $(50 \%)$ of 12 children, this was not statistically significant.

An additional finding was that children with functional defects of the valve tended to show higher rates of regression than the children with structural changes. The 72 cases of borderline or definite RHD were stratified into two subgroups based on the presence or the absence of MR based on the WHF criteria and compared in relation to progression of valve lesions. Of the 57 cases with MR, $9(16 \%)$ had progression of their disease (of which 2 progressed sufficiently to be categorised as having definite RHD) compared with 5 (33\%) out of 15 cases who did not have MR (and whose valvular abnormalities were structural). However, this was not statistically significant $(\mathrm{P}=0.086)$.

\section{DISCUSSION}

This study demonstrates a high prevalence of subclinical RHD in schoolchildren in Aswan. The overall prevalence of 31 cases per 1000 children is high. It is comparable with similar studies of urban/periurban schoolchildren in Jimma, Ethiopia and Lilongwe, Malawi (31 per 1000 and 34 per 1000 , respectively) ${ }^{1718}$ and higher than that found in children in Cape Town, South Africa and Kampala, Uganda (20 and 15 cases per 1000 , respectively). ${ }^{14} 17$ The ratio of definite to borderline disease was also higher (1.7) than that observed in these other studies which ranged from 0.3 in South Africa to 1.5 in Ethiopia suggesting a more aggressive pattern of disease. The prevalence rates using the current WHF criteria give estimates which are much higher than previously reported data from Egypt based on clinical examination $(0.7 / 1000)$ or older echocardiographic studies $(6.2 / 1000) .{ }^{19} 20$ The high rates are supported by the very high prevalence of clinical disease, where at the newly established Aswan Heart Centre it represents $50 \%$ of the medical and $20 \%$ of the surgical workload.

The study was based on the screening of a large number of children who were carefully selected to be representative of the school-age population of Aswan. Although the use of school children may underestimate prevalence due to non-attendance, ${ }^{21}$ this is unlikely to have biased the current study as school attendance is known to be high in Aswan (approximately 96\%). The echocardiographic diagnosis of RHD was revised by two experienced cardiologists and categorised according to the current WHF criteria. The value of this technique in detecting subclinical disease is underlined by the very low (7\%) sensitivity of clinical auscultation. An important and novel aspect is the follow-up which was carried out a mean of 42 months after the original study. Although a follow-up rate of $76 \%$ was achieved, it is possible that the non-responding children could have had a more adverse progression of their RHD as they were less likely to have been compliant with the penicillin prophylaxis. However, when contacted, the parents of these children refused follow-up, stating that their children were healthy and had no signs of disease. Most of the children successfully followed up were on penicillin prophylaxis and although there was some evidence that compliance reduced the rate of progression, we did not have sufficient statistical power in the study to evaluate the effectiveness of secondary prophylaxis. The small numbers also limited our ability to do multivariate analyses of the factors which were linked with disease progression or allow for the influence of confounders.

Our findings do, however, suggest that there is considerable change in the echocardiographic appearances over this time with evidence of both regression and progression of the cardiac abnormalities. The majority 
of the children originally diagnosed as having definite disease remained unchanged; of the rest approximately equal numbers showed evidence of regression or progression. Findings shown in figure 1 were similar for the group diagnosed with borderline disease. While some of this could be attributed to observer variation in the echocardiographic diagnosis of RHD or within-patient alterations in cardiac physiology, the high level of agreement between the screening diagnosis and diagnosis following expert review suggests that this cannot explain the changes observed during the follow-up study. It is more consistent with the waxing and waning of the inflammatory changes underlying RHD which is a known feature of many autoimmune diseases.

There have been few studies examining the progression of subclinical RHD detected in echocardiographic studies and comparison is difficult because of the different disease categorisations and follow-up intervals. Our conclusion, which are limited by the relatively small numbers, accord with studies carried out in Australian Aborigines ${ }^{13}$ and in Africa ${ }^{14} 16$ which all suggest that both progression and regression of valve lesions are a feature of RHD. Among 55 Aborigines with borderline disease, 13 (23\%) progressed to definite disease after an interval of between 2.5 and 5 years. ${ }^{13}$ Findings were similar in the 2-year follow-up of Ugandan schoolchildren were among the 43 cases followed up, 18 improved and 4 worsened, ${ }^{14}$ and in the South African study of 44 borderline and definite cases of RHD followed up at 5-yr showed that 21 had become normal, 2 were improved, 14 were unchanged and 7 progressed from borderline to definite disease. ${ }^{16}$ These studies also reported that progression was related to affection of the MV rather than the AV and suggest that both functional and morphological changes of the MV are important in detecting those at greatest risk of progression of RHD. ${ }^{13}$ Our study suggests the possibility that functional affection and the absence of structural pathology is a good prognostic sign for regression. We also found that disease regression was less likely to occur in older children.

Importantly, this study serves to outline the importance of RHD as a major public health issue which needs to be addressed initially by establishing prospective RHD registers and multisectoral and integrated national RHD control programme. These should ensure proper screening for signs of RHD, developing appropriate expertise in the diagnosis of ARF and RHD, implementation of secondary prevention and ensuring adequate penicillin prophylaxis. Finally, stimulating efforts to evolve effective primary prevention methods such as vaccines remains a priority.

Acknowledgements The authors would like to thank AHC staff for their contribution and assistance with sample collection and follow-up. They also thank the schools and their personnel and most importantly all children and their families in the cohort for their compliance to the visits.
Contributors SK: carried out the echocardiographic screening; responsible for the overall content. KS and AA: reviewed the echocardiograms. SK, DIP and HM: carried out the analysis. SK, DIP and MY: wrote the manuscript. All authors: read and approved the final version.

Funding The study was funded by Aswan Heart Centre.

Competing interests None declared.

Patient consent Guardian consent obtained.

Ethics approval Ministry of Health.

Provenance and peer review Commissioned; internally peer reviewed.

Data sharing statement Data will be made available upon request.

Open Access This is an Open Access article distributed in accordance with the Creative Commons Attribution Non Commercial (CC BY-NC 4.0) license, which permits others to distribute, remix, adapt, build upon this work non-commercially, and license their derivative works on different terms, provided the original work is properly cited and the use is non-commercial. See: http://creativecommons.org/ licenses/by-nc/4.0/

C Article author(s) (or their employer(s) unless otherwise stated in the text of the article) 2017. All rights reserved. No commercial use is permitted unless otherwise expressly granted.

\section{REFERENCES}

1. Remenyi B, ElGuindy A, Smith SC, et al. Valvular aspects of rheumatic heart disease. Lancet 2016;387:1335-46.

2. Carapetis JR, Beaton $A$, Cunningham MW, et al. Acute rheumatic fever and rheumatic heart disease. Nat Rev Dis Primers 2016;2:15084.

3. Engelman D, Kado JH, Reményi B, et al. Focused cardiac ultrasound screening for rheumatic heart disease by briefly trained health workers: a study of diagnostic accuracy. Lancet Glob Health 2016;4:e386-394.

4. Roberts KV, Maguire GP, Brown A, et al. Rheumatic heart disease in Indigenous children in northern Australia: differences in prevalence and the challenges of screening. Med J Aust 2015;203:221.

5. World Heart Federation. Rheumatic heart disease. Geneva, Switzerland. https://www.world-heart-federation.org/programmes/ rheumatic-heart-disease

6. Reményi B, Wilson N, Steer A, et al. World Heart Federation criteria for echocardiographic diagnosis of rheumatic heart disease-an evidence-based guideline. Nat Rev Cardiol 2012;9:297-309.

7. Marijon E, Mirabel M, Celermajer DS, et al. Rheumatic heart disease. Lancet 2012;379:953-64.

8. Carapetis JR, Hardy M, Fakakovikaetau T, et al. Evaluation of a screening protocol using auscultation and portable echocardiography to detect asymptomatic rheumatic heart disease in tongan school children. Nat Clin Pract Cardiovasc Med 2008:5:411-7.

9. Marijon E, Ou P, Celermajer DS, et al. Prevalence of rheumatic heart disease detected by echocardiographic screening. $N$ Engl $J$ Med 2007:357:470-6.

10. Australian Insititute of Health and Welfare. Rheumatic heart disease: all but forgotten in Australia except among Aboriginal and Torres Strait Islander peoples. Canberra : AlHW, 2004. Bull no 16 AlHW Cat No AUS 48.

11. Paar JA, Berrios NM, Rose JD, et al. Prevalence of rheumatic heart disease in children and young adults in Nicaragua. Am J Cardiol 2010;105:1809-14.

12. Marijon E, Celermajer DS, Tafflet M, et al. Rheumatic heart disease screening by echocardiography: the inadequacy of World Health Organization criteria for optimizing the diagnosis of subclinical disease. Circulation 2009;120:663-8.

13. Rémond $M$, Atkinson $D$, White $A$, et al. Are minor echocardiographic changes associated with an increased risk of acute rheumatic fever or progression to rheumatic heart disease? Int J Cardiol 2015;198:117-22.

14. Beaton A, Okello E, Aliku T, et al. Latent rheumatic heart disease: outcomes 2 years after echocardiographic detection. Pediatr Cardiol 2014;35:1259-67.

15. Zühlke L, Mayosi BM. Echocardiographic screening for subclinical rheumatic heart disease remains a research tool pending studies of impact on prognosis. Curr Cardiol Rep 2013;15:343.

16. Zühlke L, Engel ME, Lemmer $C E$, et al. The natural history of latent rheumatic heart disease in a 5 year follow-up study: a prospective 
observational study. BMC Cardiovasc Disord

2016;16:46

17. Engel ME, Haileamlak A, Zühlke L, et al. Prevalence of rheumatic heart disease in 4720 asymptomatic scholars from South Africa and Ethiopia. Heart 2015;101:1389-94.

18. Sims Sanyahumbi A, Sable CA, Beaton A, et al. School and community screening shows Malawi, Africa, to have a high prevalence of latent rheumatic heart disease. Congenit Heart Dis 2016;11:615-21.
19. El-Aroussy W, El-Hagracy N, Fawzy H, et al. Prevalence of rheumatic valvular heart disease among Egyptian school children: an echocardiographic screening. Med J Cairo Univ 2013;81:139-44.

20. Abdel-Moula AM, Sherif AA, Sallam SA, et al. Prevalence of rheumatic heart disease among school children in Alexandria, Egypt: a prospective epidemiological study. J Egypt Public Health Assoc 1998;73:233-54.

21. Gemechu T, Mahmoud H, Parry EH, et al. Community-based prevalence study of rheumatic heart disease in rural Ethiopia. Eur $J$ Prev Cardiol 2017;24:717-23. 